\title{
Topical anti-inflammatory potential of quercetin in lipid-based nanosystems: in vivo and in
}

\section{vitro evaluation}

Carla Caddeo $^{\mathrm{a}, *}$, Octavio Díez-Sales ${ }^{\mathrm{b}, \mathrm{c}}$, Ramon Pons ${ }^{\mathrm{d}}$, Xavier Fernàndez-Busquets ${ }^{\mathrm{e}, \mathrm{f,g}}$, Anna Maria Fadda $^{\mathrm{a}}$, Maria Manconi ${ }^{\mathrm{a}}$

${ }^{a}$ Dept. of Scienze della Vita e dell'Ambiente, Sezione di Scienze del Farmaco, University of Cagliari, Via Ospedale 72, 09124 Cagliari, Italy

${ }^{\mathrm{b}}$ Dept. of Pharmacy and Pharmaceutical Technology, University of Valencia, Avda Vicente Andrés Estellés s/n, 46100-Burjassot, Valencia, Spain

c Instituto de Reconocimiento Molecular y Desarrollo Tecnológico, Centro Mixto Universidad Politécnica de Valencia-Universidad de Valencia, Spain

${ }^{\mathrm{d}}$ Dept. of Tecnologia Química i de Tensioactius, Institut de Química Avançada de Catalunya (IQAC-CSIC), 08034 Barcelona, Spain

e Nanobioengineering Group, Institute for Bioengineering of Catalonia, Baldiri Reixac 10-12, Barcelona E08028, Spain.

${ }^{\mathrm{f}}$ Barcelona Centre for International Health Research (CRESIB, Hospital Clínic-Universitat de Barcelona), Rosselló 149-153, Barcelona E08036, Spain.

${ }^{\mathrm{g}}$ Biomolecular Interactions Team, Nanoscience and Nanotechnology Institute (IN $\left.{ }^{2} \mathrm{UB}\right)$, University of Barcelona, Martí i Franquès 1, Barcelona E08028, Spain

* Corresponding author:

Carla Caddeo

Dept. of Scienze della Vita e dell'Ambiente, Sezione di Scienze del Farmaco, University of Cagliari, Via Ospedale 72, 09124 Cagliari, Italy.

Tel.: +39 0706758582; fax: +39 0706758553. E-mail address: caddeoc@unica.it 


\section{ABSTRACT}

Purpose To develop quercetin-loaded phospholipid vesicles, namely liposomes and PEVs (Penetration Enhancer-containing Vesicles), and to investigate their efficacy on TPA-induced skin inflammation.

Methods Vesicles were made from a mixture of phospholipids, quercetin and polyethylene glycol 400 (PEG), specifically added to increase drug solubility and penetration through the skin. Vesicle morphology and self-assembly were probed by Cryo-Transmission Electron Microscopy and Small/Wide Angle X-ray Scattering, as well as the main physico-chemical features by Light Scattering.

The anti-inflammatory efficacy of quercetin nanovesicles was assessed in vivo on TPA-treated mice dorsal skin by the determination of two biomarkers: oedema formation and myeloperoxidase activity. The uptake of vesicles by $3 \mathrm{~T} 3$ fibroblasts was also evaluated.

Results Small spherical vesicles were produced. Their size and lamellarity was strongly influenced by the PEG content $(0 \%, 5 \%, 10 \% \mathrm{v} / \mathrm{v})$. The administration of vesicular quercetin on TPA-inflamed skin resulted in an amelioration of the tissue damage, with a noticeable attenuation of oedema and leukocyte infiltration, especially using 5\% PEG-PEVs, as also confirmed by confocal microscopy. In vitro studies disclosed a massive uptake and diffusion of PEVs in dermal fibroblasts.

Conclusions The proposed approach based on quercetin vesicular formulations may be of value in the treatment of inflammatory skin disorders.

Keywords: vesicle; quercetin; skin inflammation; mice; dermal fibroblasts 


\section{INTRODUCTION}

During last years there has been a great scientific interest in the use of phytodrugs from plant foods to treat inflammation, which is responsible for chronic wounds and cancer. In particular, dietary polyphenols are being extensively studied for their striking antioxidant, anti-inflammatory, chemopreventive and chemotherapeutic abilities (1-3). Quercetin (QUE) is one such dietary polyphenol, commonly found in fruits and vegetables like apples, berries and onions. More precisely, QUE belongs to the flavonoid class $(4,5)$. It has been shown to exert multiple pharmacological effects, such as scavenging of oxygen radicals, prevention of lipid peroxidation, chelation of transition metals, and inhibition of inflammation (4, 6-9). This high anti-radical/antioxidant activity has been attributed to the presence of three active functional groups in QUE structure (Fig. 1): the ortho-dihydroxy (catechol) moiety in the B ring, the $\mathrm{C}_{2}-\mathrm{C}_{3}$ double bond in conjunction with a 4-oxo function and the hydroxyl substitution at positions 3,5 and $7(10,11)$. The abovementioned beneficial effects, coupled with its safety profile and natural origin, make QUE a very attractive candidate for incorporation into formulations intended for skin delivery. However, despite the demonstrated safety and efficacy of QUE, its use is hampered by poor water solubility and low skin permeability $(9,12)$. Various strategies have been attempted to improve QUE permeability through the skin with the aim of facilitating topical/transdermal delivery, which include the synthesis of ester prodrugs (13), microemulsion-based approaches (6, 14), lecithinchitosan nanoparticles (12), nanostructured lipid carriers and solid lipid nanoparticles $(8,9,15)$. Most of these studies have shown some degree of QUE permeation into the skin, but no transdermal delivery. We have recently formulated QUE in PEVs, phospholipid vesicles prepared with four different hydrophilic penetration enhancers (PEs), essential to improve QUE solubility in water thanks to their high solubilizing power: Transcutol ${ }^{\circledR} \mathrm{P}$, propylene glycol, polyethylene glycol 400 and Labrasol ${ }^{\circledR}$. All PEVs promoted QUE deposition in the three main skin strata (i.e., stratum corneum, epidermis and dermis) and transdermal permeation (4). Hence, it is apparent that the 
development of lipid-based nanosystems capable of transporting QUE across the skin is of great significance. In order to ascertain whether topically applied QUE in PEVs was actually able to exert its bioactivity in coping with oxidative stress and inflammation, in vivo tests were conducted and are presented in this paper. PEVs were prepared using polyethylene glycol 400, and their physicochemical features (i.e., size distribution, surface charge, entrapment efficiency) and lamellar assembly probed. Their efficacy and safety in skin delivery were assessed by testing their activity against common inflammatory endpoints in mice: inhibition of chemically-induced oedema and leukocyte infiltration (reflected in myeloperoxidase -MPO- activity). The confocal imaging of the treated skin was also performed. In addition, the in vitro uptake and distribution of QUE-loaded vesicles in $3 \mathrm{~T} 3$ dermal fibroblasts were evaluated.

\section{MATERIALS AND METHODS}

\section{Materials}

Lipoid S75 (S75), soybean lecithin with 70\% phosphatidylcholine (and 10\% phosphatidylethanolamine, lysophosphatidylcholine 3\% maximum, triglycerides 3\% maximum, fatty acids $0.5 \%$ maximum, tocopherol $0.1-0.2 \%$ ) was purchased from Lipoid $\mathrm{GmbH}$ (Ludwigshafen, Germany). Quercetin (QUE, 3,3',4',5,7-pentahydroxyflavone; $\geq 95 \%$ pure), phosphate buffer solution (PBS, pH 7.4) and polyethylene glycol 400 (PEG) were purchased from Sigma-Aldrich (Milan, Italy). 12-O-Tetradecanoylphorbol 13-acetate (TPA) and all other reagents, if not otherwise specified, were from Sigma (Madrid, Spain).

\section{Quercetin solubility}

QUE solubility was assessed by dispersing an excess amount of the drug in $5 \mathrm{ml}$ of PBS or PEG/PBS mixtures $(5 \%, 10 \% \mathrm{v} / \mathrm{v})$. Dispersions were kept under constant stirring for $24 \mathrm{~h}$ at $25{ }^{\circ} \mathrm{C}$ 
in a thermostated bath. After QUE excess sedimentation (18 h), the clear solutions were centrifuged for 10 minutes at $10000 \mathrm{rpm}$ and the supernatant analyzed by HPLC as described elsewhere (4).

\section{Vesicle preparation}

QUE and S75 were weighed in a glass vial and left hydrating overnight in PBS, or a mixture of PEG/PBS $(5 \%, 10 \% \mathrm{v} / \mathrm{v})$, to obtain liposomes and PEVs, respectively (Table I). The suspensions were sonicated (5 seconds on and 2 seconds off, 100 cycles; $13 \mu \mathrm{m}$ of probe amplitude) with a Soniprep 150 (MSE Crowley, London, UK). Empty liposomes and PEVs were also prepared.

\section{Vesicle characterization}

Vesicle formation and morphology were checked by cryogenic transmission electron microscopy (cryo-TEM). For the analysis, a thin aqueous film was formed by placing $5 \mu 1$ of the vesicular dispersion on a glow-discharged holey carbon grid, and then blotting the grid against filter paper. The resulting thin sample film spanning the grid holes were vitrified by plunging the grid (kept at $100 \%$ humidity and room temperature) into ethane, maintained at its melting point with liquid nitrogen, using a Vitrobot (FEI Company, Eindhoven, The Netherlands). The vitreous film was transferred to a Tecnai F20 TEM (FEI Company, Eindhoven, The Netherlands) using a Gatan cryotransfer (Gatan, Pleasanton, CA), and the sample was observed in a low-dose mode. Images were acquired at $200 \mathrm{kV}$ at a temperature of $-170 /-175{ }^{\circ} \mathrm{C}$, using low-dose imaging conditions not exceeding $20 \mathrm{e}^{-} / \AA^{2}$, with a $4096 \times 4096$ pixel CCD Eagle camera (FEI Company, Eindhoven, The Netherlands).

The average diameter, polydispersity index (P.I., a measure of the width of size distribution) and zeta potential of vesicles were determined by Dynamic and Electrophoretic Light Scattering using a Zetasizer nano-ZS (Malvern Instruments, Worcestershire, UK). Samples $(\mathrm{n}=3)$ were diluted $(1: 100)$ with PBS, or PEG/PBS $\left(5 \%, 10 \%\right.$ v/v) when appropriate, and analysed at $25{ }^{\circ} \mathrm{C}$. 
Vesicles were purified from the non-incorporated drug by dialysis. Each sample $(1 \mathrm{ml})$ was loaded into Spectra/Por ${ }^{\circledR}$ tubing (12-14 kDa MW cut-off; Spectrum Laboratories Inc., DG Breda, The Netherlands) and dialyzed against PBS (2 L), or PEG/PBS (5\%, 10\% v/v) when appropriate, which was refreshed every hour during 4 hours, at $5{ }^{\circ} \mathrm{C}$. Drug entrapment efficiency (EE\%), expressed as the percentage of the amount of drug initially used, was determined by HPLC after disruption of unpurified and purified vesicles with $0.025 \%$ Triton X-100. QUE content was assayed as described elsewhere (4).

The lipid content of the vesicle dispersions was assessed by the Stewart assay (16). Briefly, an aliquot of liposome and PEV suspension was added to a biphasic mixture of aqueous ammonium ferrithiocyanate solution $(0.1 \mathrm{~N})$ and chloroform. The concentration of S75 was obtained by measuring the absorbance at $485 \mathrm{~nm}$ into the organic phase. The aggregation efficiency (AE\%) represented the effective amount of aggregated phospholipids expressed as the percentage of the amount initially used.

\section{X-ray diffraction}

Vesicle structure was studied by Small and Wide-Angle X-ray Scattering (SWAXS). SAXS and WAXS patterns were recorded simultaneously using a S3-MICRO (Hecus X-ray systems, Graz, Austria) coupled to a GENIX-Fox 3D X-ray source (Xenocs, Grenoble, France) working at $50 \mathrm{Kv}$ and $1 \mathrm{~mA}$. This source produces a focused X-ray beam with $\lambda=1.542 \AA$ at $\mathrm{Cu} \mathrm{K}_{\alpha}$-line with more than $97 \%$ purity and less than $0.3 \% \mathrm{~K}_{\beta}$. The two detectors are position sensitive detectors PSD 50 (Hecus, Graz, Austria). The working $q$-range were $0.01 \leq q \leq 0.6 \AA^{-1}$, in the small angle, and $2.8 \leq$ $q \leq 3.5 \AA^{-1}$ in the wide angle range, where $q=(4 \pi \sin \theta) / \lambda$ is the modulus of the scattering wave vector, $\theta$ the scattering angle and $\lambda$ the wavelength. The vesicular dispersions were loaded into glass capillaries and the diffraction patterns were recorded at $25{ }^{\circ} \mathrm{C}$. All the scattering curves were reproduced twice, with subsequent calculation of the electron distance distribution, and yielded 
identical results. For the figures, a representative curve was selected, plotting the scattering intensity $I$ as a function of the scattering vector $q$. SAXS patterns were analyzed using the MCG model developed by Pabst et al. (17). The analysis technique models the full $q$-range in the SAXS regime, including Bragg peaks and diffuse scattering. The bilayer electronic density profile is calculated as the sum of three gaussians, one corresponding to the methyl contributions and the other two corresponding to the polar heads. Differently from the model by Pabst et al. (17), we also included a constant term through the hydrophobic part of the bilayer to give account of the methylene contribution. The interbilayer ordering is taken into account considering the modified Caillé model as in the work by Pabst et al. (17). By this procedure, relevant structural parameters, as well as the distribution of electron density in the polar and apolar regions of membranes, were obtained. The model allowed fitting the SAXS pattern of bilayer-based structures, i.e. vesicles and lamellar phases. From the analysis, the membrane thickness was obtained through the definition $d_{b}=2\left(Z_{h}+\right.$ fwhm / 2), where $Z_{h}$ is the distance to the center of the bilayer of the polar head Gaussian with the corresponding full-width at half maximum fwhm. To derive the other parameters, we used 159.0 electrons for the polar head and 261.9 electrons for the hydrophobic tails (based on the composition of S75). The electron density of the medium with different concentrations of PEG slightly differed from that of water. Hence, we kept the water level as a reference throughout, this having no effect on the fitting.

\section{Mice and in vivo experimental design}

Female CD-1 mice (5-6 weeks old, 25-35 g) were obtained from Harlan Laboratories (S. Pietro al Natisone, Italy) and acclimatized for 1 week before use. Animals were fed rodent pellets (Global diet 2014, Harlan Teklad, Barcelona, Spain) ad libitum with free access to water, and maintained at $20 \pm 2{ }^{\circ} \mathrm{C}$ with a relative humidity of $60 \pm 5 \%$ and a 12-h light/dark cycle. All studies were performed in accordance with European Union regulations for handling and use of laboratory 
animals. Protocols were approved by the Institutional Animal Care and Use Committee of the University of Valencia.

The back skin of mice ( $\mathrm{n}=4$ per group) was shaved one day before the experiment, and those animals showing no hair re-growth were used. TPA dissolved in acetone ( $243 \mu \mathrm{M} ; 3 \mu \mathrm{g} / 20 \mu \mathrm{l})$ was applied to the shaved dorsal area (approximately $2 \mathrm{~cm}^{2}$ ) to induce cutaneous inflammation (day 1). Negative control mice received acetone only $(20 \mu \mathrm{l})$. Empty or QUE-loaded vesicles, and QUE in a PEG/PBS mixture (10\% v/v), were applied dropwise $(20 \mu \mathrm{l})$ and carefully smeared over the same dorsal site $3 \mathrm{~h}$ after TPA application (non-occlusive conditions), until complete absorption. The procedure was repeated (at 24-h intervals) on day 2 and 3. On day 4, mice were sacrificed by cervical dislocation.

\section{Oedema formation and myeloperoxidase determination}

The inhibitory effect of vesicular QUE on TPA-induced inflammation was evaluated by two biomarkers: oedema formation and MPO activity. Mice were sacrificed after 72 hours of treatment (on day 4). The treated dorsal skin area of each mouse was excised and weighted to assess any increase indicative of oedema formation, and immediately stored at $-80{ }^{\circ} \mathrm{C}(18,19)$.

MPO assay was performed following the methods of De Young et al. (20) and Sato et al. (21), adapted to a 96-well plate format, as previously reported (19). Briefly, skin biopsies were homogenized and centrifuged. The supernatant was incubated with hydrogen peroxide and tetramethylbenzidine and then assayed for MPO activity spectrophotometrycally at $620 \mathrm{~nm}$. The MPO activity was calculated from the linear portion of a standard curve.

\section{Confocal Laser Scanning Microscopy of mouse skin}

Confocal laser scanning microscopy (CLSM) was employed to examine the architecture, ultrastructure and potential wound healing of treated mouse skin. Mice were sacrificed after 72 
hours of treatment (on day 4). The treated dorsal skin area of each mouse was excised and rapidly frozen at $-80{ }^{\circ} \mathrm{C}$. Sections of skin $(7 \mu \mathrm{m}$ thickness) were cut with a cryostat (Leica CM1950, Barcelona, Spain) orthogonally (in the $\mathrm{z}$ axis) to the surface, and examined under a FluoView FV1000 inverted confocal microscope (Olympus, Barcelona, Spain) equipped with a Ultraviolet/Visible light laser. Using a UPlanSApo 20x objective NA 0.75, images with a field size of $1024 \times 1024 \mu \mathrm{m}$ were generated.

\section{Cell culture and treatment with colloidal dispersions}

3 T3 mouse fibroblasts (ATCC collection) were grown as adherent cultures in phenol red-free Dulbecco's Modified Eagle's medium (DMEM) with high glucose, supplemented with 10\% (v/v) fetal bovine serum, penicillin $(100 \mathrm{U} / \mathrm{ml})$, and streptomycin $(100 \mu \mathrm{g} / \mathrm{ml})$ (Life Technologies, Monza, Italy), and incubated at $37^{\circ} \mathrm{C}$ in a humidified atmosphere of $5 \% \mathrm{CO}_{2}$.

Cellular uptake of the testing formulations was investigated by CLSM using vesicles labelled with fluorescent rhodamine (1,2-dioleoyl-sn-glycero-3-phosphoethanolamine- $\mathrm{N}$-(lissamine rhodamine B sulfonyl); Avanti Polar Lipids, Alabama), and loaded with fluorescent QUE. 3T3 cells were grown to confluence in 35-mm dishes and then incubated with rhodamine-labelled, QUE-loaded vesicles for 30 minutes, 2 and 4 hours. For the observation under the FluoView FV1000 confocal microscope, cells were fixed with $4 \%$ paraformaldehyde and stained with the blue-fluorescent DNA stain Hoechst 33258 (Sigma-Aldrich, Milan, Italy) to visualize the cell nucleus, and green phalloidin fluorescein-isothiocyanate for the actin cytoskeleton.

Pictures were taken in z-stacks using the 60x objective.

\section{Statistical analysis of data}

Results are expressed as the mean \pm standard deviation. Parametric and non-parametric analysis of variance (ANOVA and Kruskal-Wallis, respectively) and Bartlet's test for homogeneity of variance 
were performed using SPSS version 17.0 for Windows (SPSS Inc., USA). Post hoc testing $(P<0.05)$ for multiple comparisons was performed by Scheffe and Dunnett's T3 tests.

\section{RESULTS}

\section{Vesicle characterization: physico-chemical and structural features}

Light Scattering results, summarized in Table II, show an increase $(P<0.05)$ in the average diameter of the prepared vesicles, as a function of the presence of loaded QUE and PEG (the higher its concentration, the larger the vesicles). Nevertheless, the vesicles were small in size, between 100 and $150 \mathrm{~nm}$, with acceptable and repeatable homogeneity (P.I. $\leq 0.35$ ), and zeta potential values slightly negative $(-10 \mathrm{mV})$, due to the low charge carried by S75. PEG-PEVs loading QUE were previously prepared, but they differed in composition: a different commercial mixture of phospholipids, $\quad$ Phopholipon ${ }^{\circledR} 50 \quad$ (P50; containing $45 \%$ phosphatidylcholine, $10-18 \%$ phosphatidylethanolamine, 37\% polar lipids, and 3\% triglycerides), was used, at a 3-fold lower concentration $(60 \mathrm{mg} / \mathrm{ml})$; PEG was mixed with water at a much higher concentration $(40 \% \mathrm{v} / \mathrm{v})$; QUE concentration was 5-fold lower $(2 \mathrm{mg} / \mathrm{ml})$. Vesicles of approximately $190 \mathrm{~nm}$ in diameter were obtained, with a P.I. $\approx 0.3$ and zeta potential around $-50 \mathrm{mV}$. Such higher surface charge is attributable to the different phospholipid mixture in the different dispersion medium. Interestingly, the formulations presented in this work disclose similar features, despite loading a higher amount of QUE and containing a lower amount of PEG. The presence of PEG increased both drug solubility (by 3.5- and 5.1-fold with 5\% and 10\% PEG, respectively; Table III) and the entrapment efficiency of vesicles. This was especially true in the case of 5\%PEG-PEVs: $75 \%$ vs $52 \%$ for liposomes (Table II).

According to aggregation efficiency data from Stewart assay, around 70\% of phospholipids (Table II) were aggregated to form vesicles. 
Cryo-TEM observations confirmed the formation of small and fairly spherical vesicles, with different lamellarity, directly proportional to PEG content. There was no evidence of free QUE crystals. Figure 2 illustrates a heterogeneous population of concentric oligolamellar and multilamellar structures of approximately $150 \mathrm{~nm}$ in diameter, a value consistent with those determined by size measurements. Some invaginated vesicles were present, probably due to the occurrence of ionic gradients during sample processing.

Further insight into vesicle morphology and lamellar arrangement were gained by SAXS and WAXS, well-established techniques for the study of self-assembling nanostructures. The SAXS experimental data were fitted to a bilayer model with interbilayer correlations (see the experimental part for details). In Figure 3 the resulting fits are shown as lines. The reduced $\chi^{2}\left(\chi^{2}=1\right.$ for purely statistical noise) values were below 2, indicative of nearly perfect fitting.

Figure 3A shows the scattering patterns of liposomes and PEVs with different PEG content, together with the best fits of the modified Caille model. It is apparent that the higher the PEG content, the better defined the correlation peaks are. This implies an increase in the number of correlated bilayers $(\mathrm{N})$ and an increase in bilayer rigidity, typical of multilamellar vesicles, as reflected in the values of the fitted parameters listed in Table IV. The marked reduction of the Caillé parameter $(\eta)$ and the increase in the number of correlated lamellae $(\mathrm{N})$ are related to the increase in bilayer stiffness. Some displacements of the scattering peaks to higher $q$ values are also evident, resulting in an overall reduction of the repetition distance (d), as PEG content increased. Getting in the details of the fittings, it can be observed that this reduction of the repetition distance is governed mainly by the reduction of the water layer thickness $\left(d_{w}\right)$, while the rest of the parameters $\left(d_{c}, d_{b}\right.$ and $Z_{\mathrm{h}}$ ) show variations within the fitting uncertainty.

Comparing Figure 3B with Figure 3A and the relative fitting parameters, it can be inferred that the effect of QUE on the scattering patterns is marginal, and consequently on the bilayer characteristics, 
if compared to that of PEG. Nevertheless, in QUE-loaded vesicles (Figure 3B) the diffraction peaks are sharper, indicating a disordering effect caused by the drug.

As expected, WAXS spectra (data not reported) exhibited a broad band centered at $18 \mathrm{~nm}^{-1}$, typical of the liquid crystalline $\mathrm{L}_{\alpha}$ state of the bilayer. There were no significant differences among the samples, for either empty or QUE-loaded vesicles.

\section{In vivo inflammatory response}

TPA-induced inflammation in mice is one of the most commonly used models of inflammation for evaluating drug anti-inflammatory activity. The topical application of TPA stimulates inflammatory reactions that are mediated by leukocyte infiltration. An early hallmark of skin irritation and local inflammation is thickening within 1-4 hours due to increased vascular permeability, oedema and swelling in the dermis. Then, leukocytes migrate to the dermis within about $24 \mathrm{~h}$, and release MPO, a marker enzyme of neutrophil granules, which can be a gauge of neutrophil activation (19). In the present study, the weight of treated skin was measured as a parameter of oedema, and MPO as an index of inflammatory cell proliferation.

Topically applied liposomes and PEVs, empty and loaded with QUE, were tested for antiinflammatory efficacy, in comparison with a drug dispersion. When viewed macroscopically, TPAexposed skin appeared irritated, reddened, scaly and crusted (Figure 4). On the other hand, a remarkable amelioration of TPA damage was observed upon administration of QUE-loaded vesicles, especially 5\%PEG-PEVs: the skin was less dry and the irritation superficial (Figure 4).

Results from oedema and MPO tests confirmed these observations. TPA-induced oedema was reduced by QUE vesicle formulations ( 1.7-fold, $P<0.05$; Figure 5), which were also effective in inhibiting MPO activity (Figure 5). QUE-loaded liposomes and 10\%PEG-PEVs provided a 2.5-fold decrease $(P<0.05)$ in MPO, while with 5\%PEG-PEVs a 4.7-fold decrease $(P<0.01)$ was achieved. 


\section{Confocal imaging of mouse treated skin}

Ultrastructural studies of TPA-exposed skin revealed altered epidermal tissue architecture and major alterations in dermal connective tissue, characterized by damaged and severely disorganized collagen fibers (Figure $6 \mathrm{~B}$ ). When the supporting connective tissue becomes disorganized and loosely arranged, the skin loses its flexibility, strength and firmness. On the contrary, when the vesicular formulations containing QUE were administered, the skin featured healing processes involving an abnormally large number of collagen fibers and amorphous ground substance, produced to repair the damage done to the dermis by TPA. Such tissue regeneration was particularly evident in the skin treated with QUE-loaded 5\%PEG-PEVs (Figure 6 C).

\section{Cellular uptake of vesicles}

The internalisation of vesicles by $3 \mathrm{~T} 3$ cells was studied by confocal microscopy. To this purpose, fibroblasts were incubated for 30 minutes, 2 and 4 hours with liposomes and PEVs labelled with red-fluorescent rhodamine and loaded with QUE, a fluorescent molecule. After 30 minutes, vesicles were spread into the cytoplasm, as indicated by the orange fluorescence due to the merging of redrhodamine and yellow-QUE (Figure 7). In liposome-exposed cells, the red fluorescence tended to progressively disappear after 2 and 4 hours, while the opposite phenomenon occurred when PEVs were used: an intense reddish fluorescence was observed, especially after 4 hours of treatment with both PEVs (Figure 7). Hence, a massive distribution of vesicular QUE can be concluded.

\section{DISCUSSION}

The present paper reports the development of lipid-based nanovesicles for the loading and skin delivery of QUE. Liposomes and PEVs with favourable physico-chemical and morphological features were obtained. All vesicles were small in size, spherical in shape and with high drug entrapment efficiency, especially PEVs, most likely due to the high solubilising power of PEG. 
PEG is a pharmaceutically accepted cosolvent that works by reducing the interfacial tension between water and a hydrophobic drug. Being its structure $\mathrm{H}-\left(\mathrm{O}-\mathrm{CH}_{2}-\mathrm{CH}_{2}\right)_{n}-\mathrm{OH}$, where $n$ is approximately 8 to 9, PEG has hydrophilic hydrogen-bonding groups that ensure water miscibility, and hydrophobic hydrocarbon regions that interfere with water self-association by disrupting its hydrogen bonding network. This creates a less-polar environment that results in more lipophilic drug molecules going into solution. Accordingly, PEG-PEVs yielded a much higher loading of lipophilic QUE than liposomes.

Thanks to its solubilising ability, PEG was able to modify vesicle packing and features, as revealed by Cryo-TEM and SWAXS. These techniques were employed to gain thorough structural and dynamical information about the vesicles and their interactions with host molecules (i.e., QUE and PEG). SAXS profiles displayed a strong dependence of vesicle lamellarity on the presence and concentration of PEG $(0 \%, 5 \%, 10 \%)$. Being PEG highly hydrophilic, it strongly interacts with the interlamellar water layers and the polar regions of phospholipids, thus affecting the overall vesicular structure. This alters the bilayer microstructure, with consequent changes (i.e., increase) in the number of correlated lamellae and their rigidity. With the increasing concentration of PEG, the SAXS reflections became more intensive and narrower, a finding clearly indicating increasing ordering, thus a multilamellar organization. In the light of these findings, it can be concluded that liposomes consisted of a heterogeneous population of unilamellar and oligolamellar vesicles, while PEVs were predominantly or uniquely multilamellar, as PEG content increased (i.e., 5\% and 10\%, respectively). It is noteworthy that QUE showed a marginal disordering effect on the structure of both liposome and PEV bilayers, with respect to PEG, most likely due to the localization at the bilayer-water interface. The water layer thickness increased upon drug incorporation $\left(\mathrm{d}_{\mathrm{w}}\right.$ from 2.9 to 3.0 in liposomes, from 1.9 to 2.2 in 5\%PEG-PEVs). This increase was accompanied by a small decrease in $d_{b}$, which is not due to a decrease in $d_{c}$. This may be congruent with QUE getting in the lipid-water interface, and may also explain the increase in bilayer rigidity (decrease of Caillé 
parameter). On the other hand, in PEVs, QUE locates at the bilayer-water interface as well as in the water phase, because water-miscible PEG favors the solubilization of the drug in the aqueous dispersion.

The results of vesicle lamellarity obtained by SAXS were in full agreement with Cryo-TEM examination, which also corroborated size measurements by Light Scattering, as vesicles larger than $100 \mathrm{~nm}$ were shown.

The anti-inflammatory potential of QUE in liposomes and PEVs was evaluated in vivo. Our data from a previous work established that chemically-induced inflammation on the skin was at a maximum after 72 hours of TPA exposure (19). TPA application elicits a series of reactions, namely inflammation and oxidative stress, that can be gauged by oedema formation and MPO activity. It was found that QUE decreased inflammation of the skin by reducing irritation, oedema and leukocyte accumulation. Further, as highlighted by confocal imaging, QUE triggered tissue regeneration by stimulating new tighter collagen fibers to grow and the production of ground substance, in order to restore the skin architecture disorganized by TPA. These remarkable benefits provided by nanoentrapped QUE, especially when delivered by 5\%PEG-PEVs, were supposed to be due to a synergistic mechanism between PEG and the vesicles. PEG improves vesicular bilayer fluidity and also reduces the stratum corneum barrier function transiently, thus creating an easier pathway for the highly fluidized vesicles (22-24). PEVs can enter intact (at least partially) the stratum corneum with the loaded QUE, and diffuse down to the epidermis and dermis. Here, they come into contact with fibroblasts and facilitate drug uptake, allowing it to exert its antiinflammatory/antioxidant activity where necessary (i.e., the dermis mainly). Interestingly, in vivo studies provided evidence that topically applied QUE, when delivered by 5\%PEG-PEVs, was more effective than previously tested diclofenac in PEVs at the same dose $(10 \mathrm{mg} / \mathrm{ml})$, under the same conditions (19). The major effect was on MPO activity: with QUE-loaded 5\%PEG-PEVs a 4.7-fold decrease was achieved, versus a 2.7-fold with diclofenac-loaded PEVs. Therefore, it can be deduced 
that natural QUE, in the appropriate formulation, possesses a superior efficacy in counteracting the inflammatory responses provoked by TPA on the skin. Inflammation is a multifactorial process involving the release of MPO from neutrophils and monocytes, which leads to the generation of reactive oxygen species (ROS) implicated in skin damage. The superior efficacy of QUE over diclofenac is reasonably due to its dual action: coping with ROS and increasing fibroblast proliferation. Fibroblasts are found within the dermis and are responsible for the synthesis of collagen, glycoproteins, glycosaminoglycans and other elements of the extracellular matrix of the connective tissue, which provides mechanical support to skin. Hence, dermal fibroblasts play a key role in wound healing: without them, the wound site could not regenerate extracellular matrix and epidermis skin cells could not proliferate over the wound site, and the skin could not properly recover from injury. In vitro studies showed that QUE-loaded PEVs were able to easily enter the fibroblasts and massively diffuse in the cytoplasm, at least for 4 hours, thus allowing QUE to exert its actions. Conversely, liposomes were rapidly internalized into the cell interior, where their content is released subsequent to vesicle degradation by lysosomal and endosomal enzymes. This different behaviour explains the higher efficacy of PEVs to carry and localize QUE in the site of inflammation.

\section{CONCLUSIONS}

A comprehensive characterization of nanovesicular systems for the loading and skin delivery of QUE was carried out. In vivo results indicated that vesicles, and especially PEVs, were capable of localizing the drug in the site of inflammation, that is the dermis, inhibiting oxidative stress and leukocyte accumulation and stimulating the repair of skin damage induced by TPA. The actual entry and diffusion in dermal fibroblasts was demonstrated in vitro. In conclusion, this work shows the interesting potential of vesicular systems, particularly PEVs, in the treatment of inflammatory skin disorders. 


\section{ACKNOWLEDGMENTS AND DISCLOSURES}

This study was supported by a grant from the University of Valencia (Relacions Internacionals I Cooperació), a grant from MIUR, Italy (PRIN 2010-2011, Prot. 2010H834LS_004), a grant from the Ministerio de Ciencia e Innovación, Spain (BIO2011-25039), which included FEDER funds, and by a grant from the Generalitat de Catalunya, Spain (2009SGR-760). Dr. C. Caddeo gratefully acknowledges Sardinia Regional Government for the financial support (P.O.R. Sardegna F.S.E. Operational Programme of the Autonomous Region of Sardinia, European Social Fund 2007-2013 Axis IV Human Resources, Objective 1.3, Line of Activity 1.3.1 "Avviso di chiamata per il finanziamento di Assegni di Ricerca". 


\section{REFERENCES}

1. Caddeo C, Teskač K, Sinico C, Kristl J. Effect of resveratrol incorporated in liposomes on proliferation and UV-B protection of cells. Int J Pharm. 2008;363:183-191.

2. Caddeo C, Manconi M, Fadda AM, Lai F, Lampis S, Diez-Sales O, Sinico C. Nanocarriers for antioxidant resveratrol: formulation approach, vesicle self-assembly and stability evaluation. Colloid Surf B-Biointerfaces. 2013;111:327-332.

3. Pando D, Caddeo C, Manconi M, Fadda AM, Pazos C. Nanodesign of olein vesicles for the topical delivery of the antioxidant resveratrol. J Pharm Pharmacol. 2013;doi: 10.1111/jphp.12093.

4. Chessa M, Caddeo C, Valenti D, Manconi M, Sinico C, Fadda AM. Effect of penetration enhancer containing vesicles on the percutaneous delivery of quercetin through new born pig skin. Pharmaceutics. 2011;3:497-509.

5. Date AA, Nagarsenker MS, Patere S, Dhawan V, Gude RP, Hassan PA, Aswal V, Steiniger F, Thamm J, Fahr A. Lecithin-based novel cationic nanocarriers (Leciplex) II: improving therapeutic efficacy of quercetin on oral administration. Mol Pharmaceutics. 2011;8:716-726.

6. Vicentini FTMC, Fonseca YM, Pitol DL, Iyomasa MM, Bentley MVLB, Fonseca MJV. Evaluation of protective effect of a water-in-oil microemulsion incorporating quercetin against UVB-induced damage in hairless mice skin. J Pharm Pharmaceut Sci. 2010;13:274-285.

7. Wagner C, Vargas AP, Roos DH, Morel AF, Farina M, Nogueira CW, Aschner M, Rocha JB. Comparative study of quercetin and its two glycoside derivatives quercetin and rutin against methylmercury (MgHg)-induced ROS production in rat brain slices. Arch Toxicol. 2010;84:89-97. 8. Chen-yu G, Chun-fen Y, Qi-lu L, Qi T, Yan-wei X, Wei-na L, Guang-xi Z. Development of a quercetin-loaded nanostructured lipid carrier formulation for topical delivery. Int $\mathrm{J}$ Pharm. 2012;430:292-298.

9. Bose S, Du Y, Takhistov P, Michniak-Kohn B. Formulation optimization and topical delivery of quercetin from solid lipid based nanosystems. Int J Pharm. 2013;441:56-66. 
10. Bors W, Heller W, Michel C, Saran M. Flavonoids as antioxidants: determination of radicalscavenging efficiencies. Methods Enzymol. 1990;186:343-355.

11. Saija A, Scalese M, Lanza M, Marzullo D, Bonina F, Castelli F. Flavonoids as antioxidant agents: importance of their interaction with biomembranes. Free Radical Biol Med. 1995;19:481486.

12. Tan Q, Liu W, Guo C, Zhai G. Preparation and evaluation of quercetin-loaded lecithin-chitosan nanoparticles for topical delivery. Int J Nanomed. 2011;6:1621-1630.

13. Montenegro L, Carbone C, Maniscalco C, Lambusta D, Nicolosi G, Ventura CA, Puglisi G. In vitro evaluation of quercetin-3-O-acyl esters as topical prodrugs. Int J Pharm. 2007;336:257-262.

14. Censi R, Martena V, Hoti E, Malaj L, Di MP. Permeation and skin retention of quercetin from microemulsions containing Transcutol ${ }^{\circledR}$ P. Drug Dev Ind Pharm. 2012;38:1128-1133.

15. Cadena PG, Pereira MA, Cordeiro RBS, Cavalcanti IMF, Barros Neto B, Pimentel CB M do Carmo, Lima Filho JL, Silva VL, Santos-Magalhães NS. Nanoencapsulation of quercetin and resveratrol into elastic liposomes. Biochim Biophys Acta. 2013;1828:309-316.

16. Stewart JC, Colorimetric determination of phospholipids with ammonium ferrothiocyanate. Anal Biochem. 1980;104:10-14.

17. Pabst G, Rappolt M, Amenitsch H, Laggner P. Structural information from multilamellar liposomes at full hydration: full $q$-range fitting with high quality x-ray data. Phys Rev E Stat Phys Plasmas Fluids Relat Interdiscip Topics. 2000;62:4000-4009.

18. De Vry CG, Valdez M, Lazarov M, Muhr E, Buelow R, Fong T, Suhasini I. Topical application of a novel immunomodulatory peptide, RDP58, reduces skin inflammation in the phorbol esterinduced dermatitis model. J Invest Dermatol. 2005;125:473-481.

19. Caddeo C, Diez Sales O, Valenti D, Ruiz Saurí A, Fadda AM, Manconi M. Inhibition of skin inflammation in mice by diclofenac in vesicular carriers: liposomes, ethosomes and PEVs. Int J Pharm. 2013;443:128-136. 
20. De Young LM, Kheifets JB, Ballaron SJ, Young JM. Edema and cell infiltration in the phorbol ester-treated mouse ear aretemporally separate and can be differentially modulated by pharmacologic agents. Agents Actions. 1989;26:335-341.

21. Sato H, Nakayama Y, Yamashita C, Uno H. Anti-inflammatory effects of tacalcitol $(1,24(\mathrm{R})(\mathrm{OH}) 2 \mathrm{D} 3, \mathrm{TV}-02)$ in the skin of TPA-treated hairless mice. J Dermatol. 2004;31:200-217.

22. Manconi M, Sinico C, Caddeo C, Vila AO, Valenti D, Fadda AM. Penetration enhancer containing vesicles as carriers for dermal delivery of tretinoin. Int J Pharm. 2011;412:37-46.

23. Manconi M, Caddeo C, Sinico C, Valenti D, Mostallino MC, Lampis S, Monduzzi M, Fadda AM. Penetration enhancer-containing vesicles: composition dependence of structural features and skin penetration ability. Eur J Pharm Biopharm. 2012;82:352-359.

24. Caddeo C, Manconi M, Valenti D, Maccioni AM, Fadda AM, Sinico C. The role of Labrasol ${ }^{\circledR}$ in the enhancement of the cutaneous bioavailability of minoxidil in phospholipid vesicles. Res $\mathbf{J}$ Pharm Technol. 2012;5:1563-1569. 


\section{FIGURES}<smiles>O=c1c(O)c(-c2ccc(O)c(O)c2)oc2cc(O)cc(O)c12</smiles>

Fig. 1. Chemical structure of quercetin.

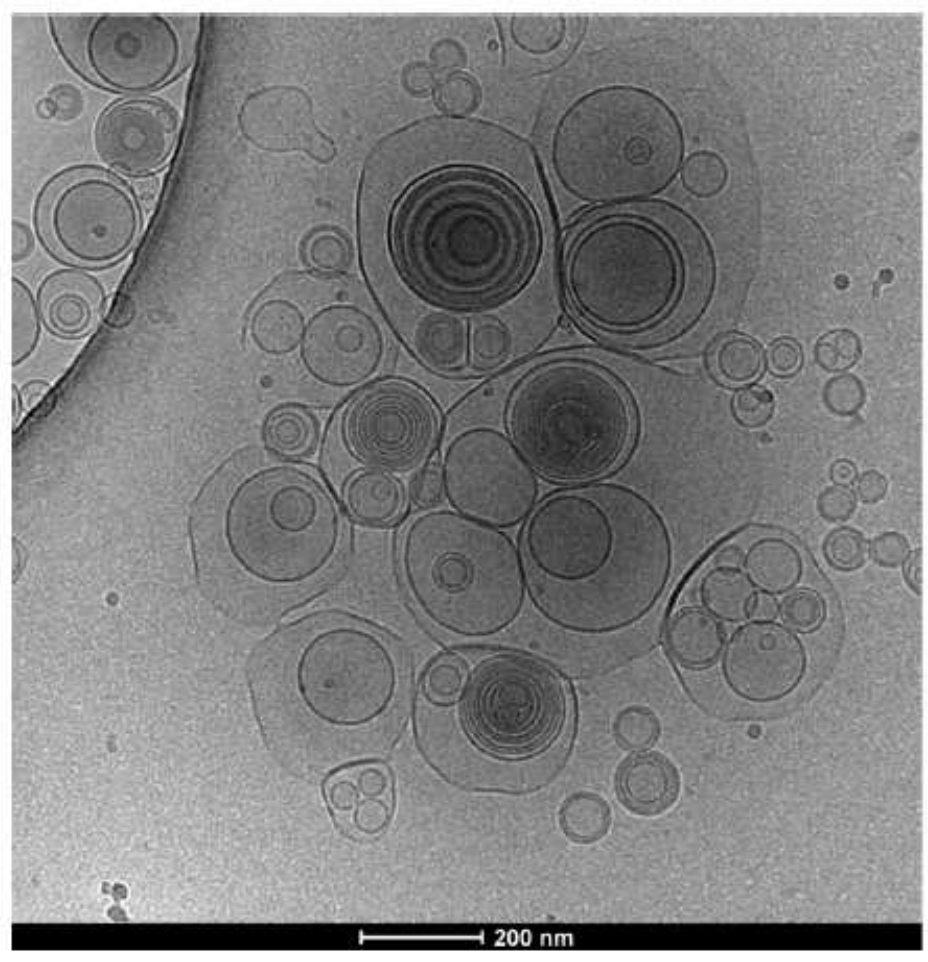

Fig. 2. Cryo-TEM image of quercetin-loaded 5\%PEG-PEVs. 

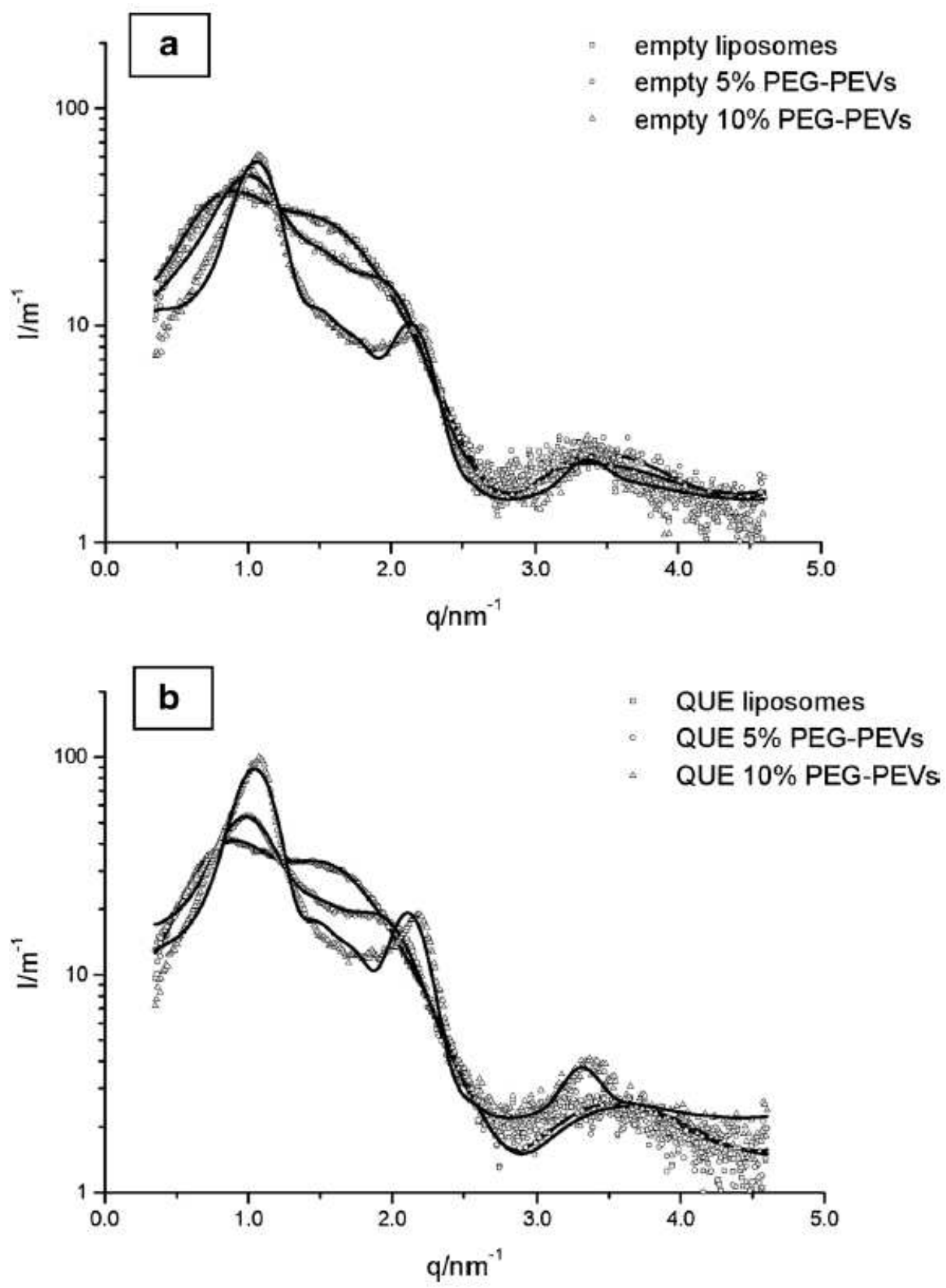

Fig. 3. SAXS patterns of empty and quercetin-loaded liposomes and PEVs. 


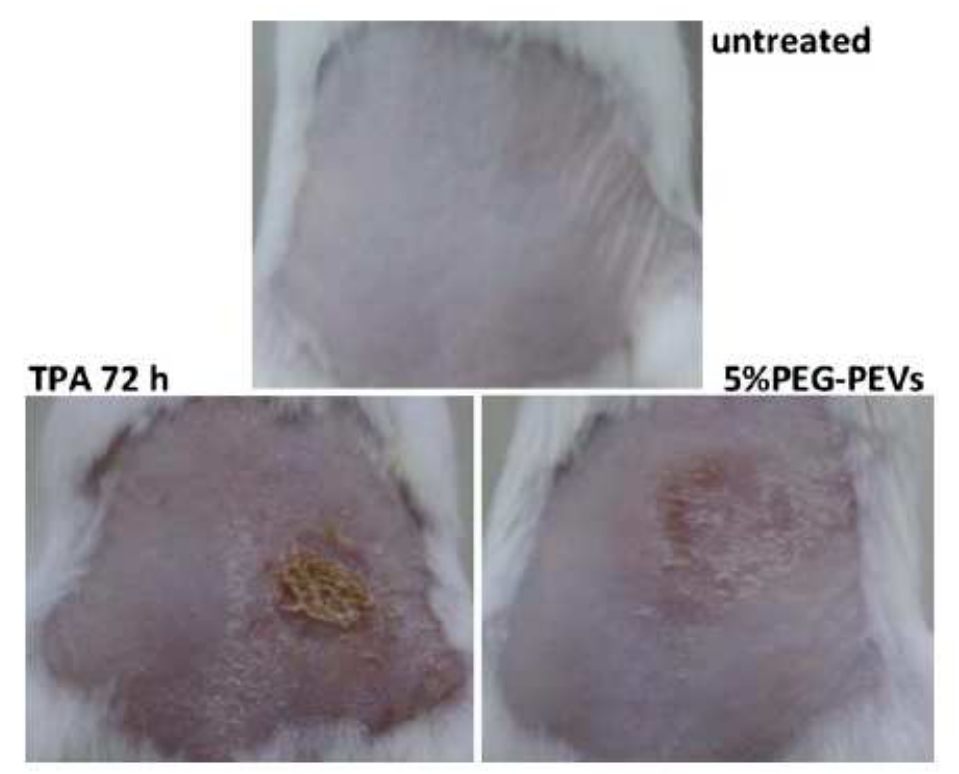

Fig. 4. Photographs of dorsal skin of mice: untreated, treated with TPA-only to induce inflammation, treated with TPA followed by the administration of quercetin-loaded 5\%PEG-PEVs.
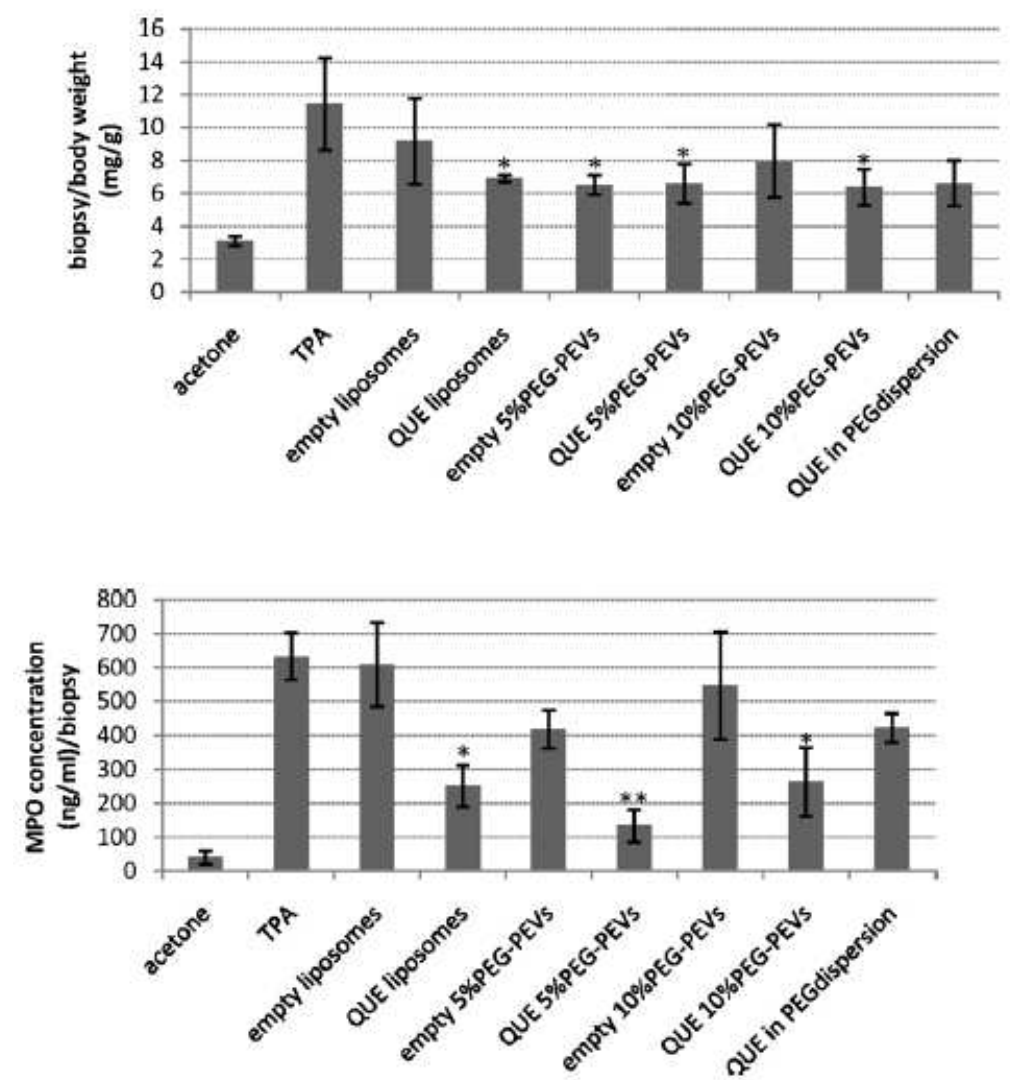

Fig. 5. Skin oedema index (skin biopsy/body weight ratio) and MPO activity of mice with TPAinduced inflammation, after treatment with testing samples, compared with TPA-only treated mice 
(positive control) and acetone receiving mice (negative control). Each bar is the mean \pm S.D. of four animals (statistical significant difference $P *<0.05, P * *<0.01$, compared to TPA).
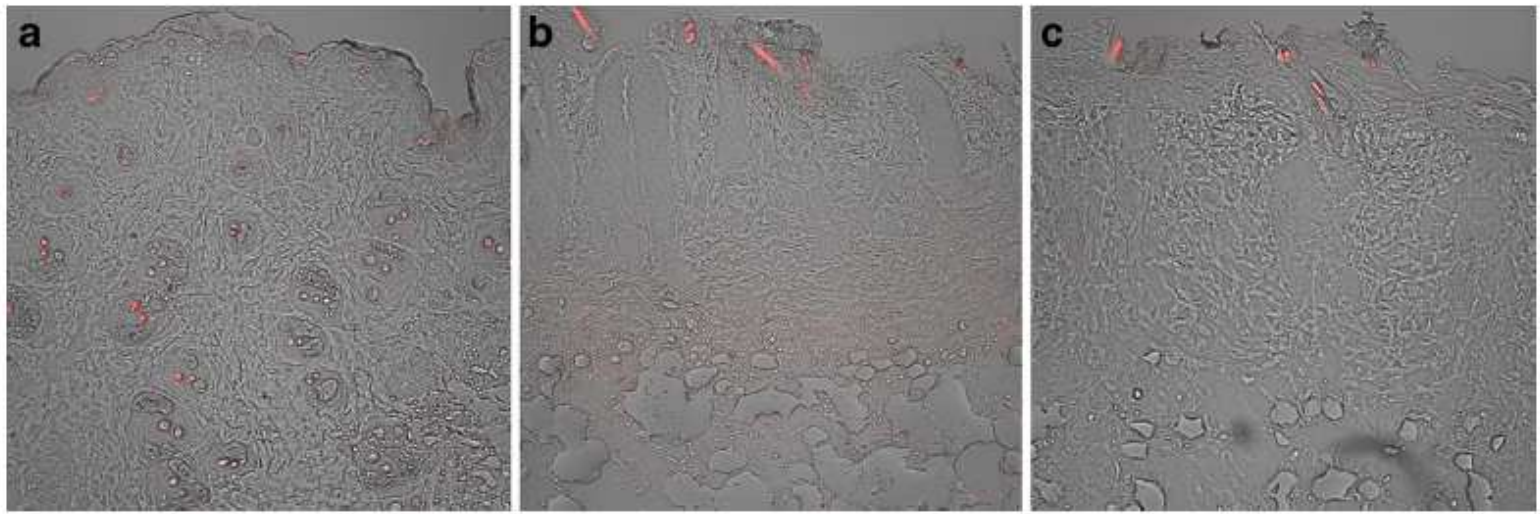

Fig. 6. Confocal cross-sectional images of mouse skin after $72 \mathrm{~h}$ of treatment with TPA-only (B), or TPA followed by the administration of quercetin-loaded 5\%PEG-PEVs (C), compared to untreated healthy skin (A). Skin autofluorescence is shown in red. 

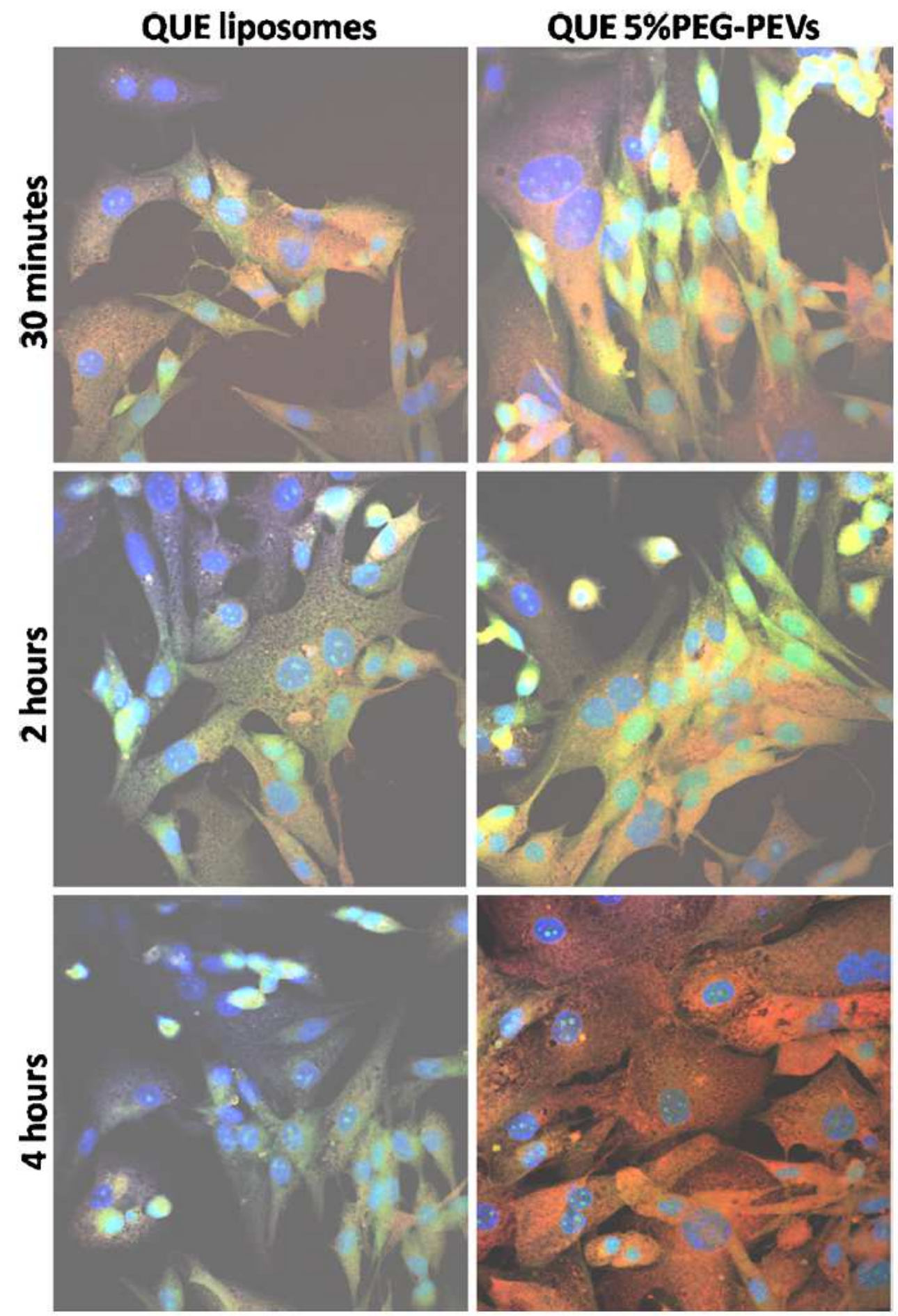

Fig. 7. 3T3 fibroblasts with blue-stained nuclei and yellow-stained actin cytoskeleton showing the progressive increase in red and yellow fluorescence as rhodamine-labelled vesicles loaded with fluorescent quercetin diffuse in the cytoplasm following a 30 minute-, $2 \mathrm{~h}$ - and $4 \mathrm{~h}$-exposure to liposomes and PEVs. 


\section{TABLES}

Table I Composition of Vesicular Formulations

\begin{tabular}{|l|l|l|l|}
\hline Component & Liposomes & $5 \%$ PEG-PEVs & $10 \%$ PEG-PEVs \\
\hline S75 & $180 \mathrm{mg}$ & $180 \mathrm{mg}$ & $180 \mathrm{mg}$ \\
\hline QUE & $10 \mathrm{mg}$ & $10 \mathrm{mg}$ & $10 \mathrm{mg}$ \\
\hline PEG & & $0.05 \mathrm{ml}$ & $0.1 \mathrm{ml}$ \\
\hline PBS & $1 \mathrm{ml}$ & $0.95 \mathrm{ml}$ & $0.9 \mathrm{ml}$ \\
\hline
\end{tabular}

Table II Characteristics of Empty and Quercetin-Loaded Vesicular Formulations: Mean Diameter,

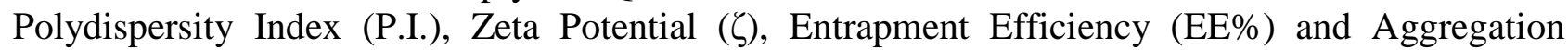
Efficiency $(\mathrm{AE} \%)$. Values are the Means \pm Standard Deviation $(n=3)$

\begin{tabular}{|l|l|l|l|l|l|l|}
\hline Sample & & $\begin{array}{l}\text { Mean diameter } \\
(\mathrm{nm})\end{array}$ & P.I. & $\zeta(\mathrm{mV})$ & EE\% & AE\% \\
\hline Liposomes & Empty & $96 \pm 2.4$ & 0.22 & $-9 \pm 0.5$ & & \\
\cline { 2 - 7 } & QUE & $116 \pm 5.3$ & 0.35 & $-9 \pm 0.4$ & $52 \pm 4.4$ & $74 \pm 4.6$ \\
\hline $\begin{array}{l}5 \% \text { PEG- } \\
\text { PEVs }\end{array}$ & Empty & $117 \pm 5.7$ & 0.23 & $-10 \pm 0.8$ & & \\
\cline { 2 - 7 } & QUE & $152 \pm 2.4$ & 0.34 & $-10 \pm 0.8$ & $75 \pm 3.0$ & $71 \pm 2.7$ \\
\hline $\begin{array}{l}10 \% \text { PEG- } \\
\text { PEVs }\end{array}$ & Empty & $136 \pm 2.6$ & 0.23 & $-9 \pm 0.8$ & & \\
\cline { 2 - 7 } & QUE & $148 \pm 3.5$ & 0.31 & $-10 \pm 0.7$ & $60 \pm 0.8$ & $73 \pm 6.3$ \\
\hline
\end{tabular}

Table III QUE Solubility in PBS or PEG/PBS Blends $(5 \%, 10 \% \mathrm{v} / \mathrm{v})$. Values are the Means \pm Standard Deviation $(n=3)$

\begin{tabular}{|l|l|}
\hline Medium & QUE $(\mu \mathrm{g} / \mathrm{ml})$ \\
\hline PBS & $9.3 \pm 0.11$ \\
\hline $5 \%$ PEG in PBS & $32.7 \pm 5.37$ \\
\hline $10 \%$ PEG in PBS & $47.5 \pm 5.20$ \\
\hline
\end{tabular}


Table IV Selected Fitting Parameters and Derived Parameters for the SAXS Curves of Liposomes and PEVs. All Distances are Given in nm. d, Repetition Distance; $\eta$, Caillé Parameter; N, Number of Correlated Lamellae; $\sigma \mathrm{h}$, Polar Head Amplitude; $\sigma c$, Methyl Amplitude; Zh, Polar Head Distance to the Center of the Bilayer; dc, Hydrophobic Chains Layer Half-Thickness; db, Bilayer Thickness; dw, Water Layer Thickness

\begin{tabular}{|l|l|l|l|l|l|l|}
\hline & $\begin{array}{l}\text { Empty } \\
\text { liposomes }\end{array}$ & $\begin{array}{l}\text { Empty } \\
5 \% \text { PEG- } \\
\text { PEVs }\end{array}$ & $\begin{array}{l}\text { Empty } \\
10 \% \text { PEG- } \\
\text { PEVs }\end{array}$ & $\begin{array}{l}\text { QUE } \\
\text { liposomes }\end{array}$ & $\begin{array}{l}\text { QUE } \\
5 \% \text { PEG- } \\
\text { PEVs }\end{array}$ & $\begin{array}{l}\text { QUE } \\
10 \% \text { PEG- } \\
\text { PEVs }\end{array}$ \\
\hline $\mathrm{d}$ & $6.94 \pm 0.5$ & $5.94 \pm 0.1$ & $5.60 \pm 0.05$ & $6.99 \pm 0.5$ & $6.03 \pm 0.1$ & $5.69 \pm 0.05$ \\
\hline$\eta$ & $0.38 \pm 0.2$ & $0.080 \pm 0.010$ & $0.038 \pm 0.005$ & $0.31 \pm 0.2$ & $0.16 \pm 0.02$ & $0.012 \pm 0.002$ \\
\hline $\mathrm{N}$ & $1.5 \pm 0.3$ & $2.5 \pm 0.3$ & $3.5 \pm 0.2$ & $1.5 \pm 0.3$ & $2.5 \pm 0.3$ & $3.5 \pm 0.2$ \\
\hline$\sigma_{\mathrm{h}}$ & $0.32 \pm 0.01$ & $0.36 \pm 0.01$ & $0.37 \pm 0.01$ & $0.31 \pm 0.01$ & $0.28 \pm 0.01$ & $0.34 \pm 0.01$ \\
\hline$\sigma_{\mathrm{c}}$ & $0.24 \pm 0.01$ & $0.20 \pm 0.01$ & $0.19 \pm 0.01$ & $0.25 \pm 0.01$ & $0.17 \pm 0.01$ & $0.25 \pm 0.01$ \\
\hline $\mathrm{Z}_{\mathrm{h}}$ & $1.63 \pm 0.02$ & $1.62 \pm 0.02$ & $1.56 \pm 0.01$ & $1.61 \pm 0.02$ & $1.57 \pm 0.02$ & $1.63 \pm 0.01$ \\
\hline $\mathrm{d}_{\mathrm{c}}$ & $1.26 \pm 0.02$ & $1.20 \pm 0.02$ & $1.12 \pm 0.02$ & $1.24 \pm 0.02$ & $1.24 \pm 0.02$ & $1.24 \pm 0.02$ \\
\hline $\mathrm{d}_{\mathrm{B}}$ & $4.02 \pm 0.05$ & $4.07 \pm 0.05$ & $4.00 \pm 0.05$ & $3.96 \pm 0.05$ & $3.81 \pm 0.05$ & $4.07 \pm 0.05$ \\
\hline $\mathrm{d}_{\mathrm{w}}$ & $2.9 \pm 0.5$ & $1.9 \pm 0.1$ & $1.6 \pm 0.1$ & $3.0 \pm 0.5$ & $2.2 \pm 0.1$ & $1.6 \pm 0.1$ \\
\hline
\end{tabular}

